Draft VERSiOn OCTOBER 16, 2018

Preprint typeset using $\mathrm{LAT}_{\mathrm{E}} \mathrm{X}$ style emulateapj v. 5/2/11

\section{SEVEN NEW CARBON-ENHANCED METAL-POOR RR LYRAE STARS} \\ Catherine R. Kennedy \\ Research School of Astronomy and Astrophysics, Australian National University, Canberra, ACT 2611, Australia
}

RiChard J. StANCLifFe

Argelander-Institut für Astronomie, Auf dem Hügel 71, 53121 Bonn, Germany and

Research School of Astronomy and Astrophysics, Australian National University, Canberra, ACT 2611, Australia

Charles KuehN

Sydney Institute for Astronomy, University of Sydney, Sydney, Australia

Timothy C. Beers

National Optical Astronomy Observatory, Tucson, AZ 85719, USA

and JINA: Joint Institute for Nuclear Astrophysics

T. D. KINMAN

National Optical Astronomy Observatory, Tucson, AZ 85719, USA

VINICIUS M. PlACCO
Gemini Observatory, Hilo, HI 96720, USA

\author{
Henrique Reggiani, Silvia Rossi \\ Departamento de Astronomia - Instituto de Astronomia, Geofísica e Ciências Atmosféricas, Universidade de São Paulo, São Paulo, SP \\ 05508-900, Brazil \\ Young Sun LeE \\ Department of Astronomy, New Mexico State University, Las Cruces, NM 88003, USA \\ Draft version October 16, 2018
}

\begin{abstract}
We report estimated carbon-abundance ratios, $[\mathrm{C} / \mathrm{Fe}]$, for seven newly-discovered carbon-enhanced metal-poor (CEMP) RR Lyrae stars. These are well-studied RRab stars that had previously been selected as CEMP candidates based on low-resolution spectra. For this pilot study, we observed eight of these CEMP RR Lyrae candidates with the Wide Field Spectrograph (WiFeS) on the ANU 2.3m telescope. Prior to this study, only two CEMP RR Lyrae stars had been discovered: TY Gru and SDSS J1707+58. We compare our abundances to new theoretical models of the evolution of lowmass stars in binary systems. These simulations evolve the secondary stars, post accretion from an AGB donor, all the way to the RR Lyrae stage. The abundances of CEMP RR Lyrae stars can be used as direct probes of the nature of the donor star, such as its mass, and the amount of material accreted onto the secondary. We find that the majority of the sample of CEMP RR Lyrae stars is consistent with AGB donor masses of around 1.5 - 2.0 $\mathrm{M}_{\odot}$ and accretion masses of a few hundredths of a solar mass. Future high-resolution studies of these newly-discovered CEMP RR Lyrae stars will help disentangle the effects of the proposed mixing processes that occur in such objects.
\end{abstract}

Subject headings: Galaxy: halo — stars: abundances — stars: variables: RR Lyrae - techniques: spectroscopic

\section{INTRODUCTION}

Over the past few decades, it has become clear that a large fraction of stars with significant carbon enhancements exists among the populations of metal-poor stars in the Galactic halo. These carbon-enhanced metalpoor (CEMP) stars were originally defined as having metallicities $[\mathrm{Fe} / \mathrm{H}] \leq-1.0$ and carbon-abundance ra-

catherine.kennedy@anu.edu.au tios $[\mathrm{C} / \mathrm{Fe}] \geq+1.0$ (Beers \& Christlieb 2005). Recent studies of large numbers of metal-poor stars suggest that a more natural dividing line between the carbon-normal and carbon-enhanced populations is $[\mathrm{C} / \mathrm{Fe}] \geq+0.7$ (see, for example, Figure 4 from Aoki et al. 2007 and Figure 4 from Carollo et al. 2012). We therefore define CEMP stars as having $[\mathrm{Fe} / \mathrm{H}] \leq-1.0$ and $[\mathrm{C} / \mathrm{Fe}] \geq+0.7$.

The frequency of CEMP stars, among metal-poor stars in the Milky Way, increases with decreasing metallicity (Beers et al. 1992; Norris et al. 1997; Beers \& Christlieb 
2005; Cohen et al. 2005: Marsteller et al. 2005: Rossi et al. 2005; Frebel et al. 2006; Lucatello et al. 2006; Norris et al. 2007; Carollo et al. 2012; Lee et al. 2013; Norris et al. 2013b; Spite et al. 2013), as well as with distance from the Galactic plane (Frebel et al. 2006; Carollo et al. 2012; Lee et al. 2013). From the study of their elemental abundance patterns, one can begin to uncover details concerning the nature of their progenitor objects.

There exist a number of different sub-classes of CEMP stars with specific abundance characteristics; these different sub-classes are suggestive of different sites of carbon production at early times (Beers \& Christlieb 2005). $\mathrm{CEMP}_{-} s$ stars, which exhibit evidence of $s$-processelement enhancement, are the most common; around $80 \%$ of CEMP stars exhibit $s$-process-element enhancements (Aoki et al. 2007), including both the CEMP-s and CEMP-r/s sub-classes (the latter sub-class indicates stars for which the presence of both $r$ - and $s$ process element enhancements are found). It is widely believed that these objects are the result of mass transfer from a companion asymptotic giant-branch (AGB) star, where the production of carbon and $s$-process elements occurs (Herwig 2005; Sneden et al. 2008). About half of the CEMP-s stars have been shown to be CEMP$r / s$, suggesting formation from molecular clouds that had already been enhanced in $r$-process elements, opening the possibility that their carbon enhancements arose from more than one site. In any case, observational evidence suggests that the CEMP-r/s stars (and other $r$-process-element rich stars) do not require a contribution of $r$-process elements from a binary companion (see Hansen et al. 2011b, 2013). Stars in the CEMP-no sub-class exhibit no neutron-capture element enhancements, and the source of the carbon enhancement for these stars is less certain. Current suggestions include the possibility that very massive, rapidlyrotating, mega metal-poor $([\mathrm{Fe} / \mathrm{H}]<-6.0)$ stars were very efficient producers of carbon, nitrogen, and oxygen, due to distinctive internal burning and mixing episodes followed by strong mass loss (Hirschi et al. 2006; Meynet et al. 2006, 2010). Another suggested origin is pollution of the interstellar medium (ISM) by so-called faint supernovae associated with the first generations of stars, which can experience extensive mixing and fallback during their explosions (Umeda \& Nomoto 2003; Tominaga et al. 2007; Kobavashi et al. 2011; Ito et al. 2013; Nomoto et al. 2013; Tominaga et al. 2013).

Recent observations of some of the lowest-metallicity RR Lyrae stars have revealed similarities between the abundance patterns of these stars and their non-varying counterparts in the halo of the Milky Way. For example, Hansen et al. (2011a) find that the elemental abundance patterns of two very metal-poor RR Lyrae stars $([\mathrm{Fe} / \mathrm{H}]$ $\sim-2.8$ ) match typical patterns of very metal-poor stars observed in the Galactic halo, and indeed, they can be compared to theoretical yields from the first generations of stars.

Because a large fraction of the very metal-poor (VMP; $[\mathrm{Fe} / \mathrm{H}]<-2.0)$ stars studied in the Galactic halo are CEMP stars, one would expect that a similar fraction of VMP horizontal-branch stars would exhibit carbon over-abundances. Prior to our observational program, only two CEMP RR Lyrae stars had been recognized
(Preston et al. 2006; Kinman et al. 2012). Now, the sample size has increased by more than a factor of four, as we have identified seven new CEMP RR Lyrae stars.

One clear advantage of the study of CEMP RR Lyrae stars, as opposed to CEMP stars in other evolutionary stages, is that the observed surface abundances are primarily influenced by the dilution of accreted material in the receiving star at first dredge up. When the receiving star is on the main sequence, it is unknown whether the observed surface composition is purely due to the makeup of the accreted material, or whether some nonconvective process (such as rotation, e.g., Masseron et al. 2012, or thermohaline mixing, e.g., Stancliffe et al. 2007) has already led to dilution. By using more evolved objects we bypass this uncertainty. Detailed stellar models (Stancliffe et al. 2013) suggest that the surface composition of a CEMP star in the RR Lyrae phase is predominantly determined by the mass of material accreted, and the composition of the ejecta. This assumes that all evolved objects had a mass of around $0.8 \mathrm{M}_{\odot}$ at the main sequence turn-off, and consequently, their structures along the giant branch (particularly the depth that the convective envelope reaches during first dredge-up) are similar. The CEMP RR Lyrae stars may thus provide a constraint on the efficiency of wind accretion, which, although currently highly uncertain (Abate et al. 2013), potentially has an important impact on, e.g., Type Ia supernova progenitors.

This paper is organized as follows. Section 2 describes the target selection, observing techniques, and details of the observations. Sections 3 and 4 describe the estimation of stellar parameters and carbon abundances. A discussion of the Oosterhoff classifications of our program stars, and their implications, is given in Section 5. Section 6 includes a description of the theoretical models used for comparison, and Section 7 contains a thorough discussion of the analysis of our measured $[\mathrm{C} / \mathrm{Fe}]$ abundances in terms of these theoretical scenarios. Our conclusions and plans for future work are provided in Section 8 .

\section{TARGET SELECTION AND OBSERVATIONS}

The RR Lyrae stars observed for this pilot program, listed in Kinman et al. (2012), were selected from the Hamburg/ESO objective-prism survey, crossmatched with previously recognized RR Lyraes. The benefit of drawing CEMP candidates from the HES is that (rather crude) preliminary estimates of $[\mathrm{Fe} / \mathrm{H}]$ and $[\mathrm{C} / \mathrm{Fe}]$ from their low-resolution spectra are available (Christlieb et al. 2008). Higher-resolution spectroscopic observations remain necessary to confirm these values, as the errors on these low-resolution estimates are quite large. Furthermore, observations of these RR Lyraes during the HES is at random phase, whereas in order to obtain accurate estimates of their atmospheric parameters (and elemental abundances) it is crucial to obtain their spectra during times of low activity.

The variable nature of RR Lyrae atmospheres poses some challenges for carrying out spectroscopic observations. The radial pulsations of these stars means that their surface gravity and effective temperature will vary significantly over short intervals of time. These pulsations also impact the star's spectral lines, causing them to shift due to the radial velocities of the gas in the outer 
Table 1

Details of Targets and Observations

\begin{tabular}{|c|c|c|c|c|c|c|c|c|c|}
\hline Star & $\begin{array}{c}\text { R.A. } \\
\text { (J2000) }\end{array}$ & $\begin{array}{c}\text { Decl. } \\
\text { (J2000) }\end{array}$ & Max Mag. & $\begin{array}{c}\text { Amplitude } \\
\text { (mag) }\end{array}$ & Waveband & $\begin{array}{c}\text { Period } \\
\text { (days) }\end{array}$ & $\begin{array}{c}\text { Ephemeris } \\
\text { Source }\end{array}$ & Phase & $\begin{array}{l}\text { Exposure } \\
(\mathrm{sec})\end{array}$ \\
\hline IV Leo & $10: 58: 12.6$ & $-00: 05: 40$ & 15.4 & 0.4 & $V$ & 0.6358 & 3 & 0.436 & 1200 \\
\hline LO Leo & $11: 35: 22.7$ & $-00: 53: 42$ & 14.8 & 1.1 & $V$ & 0.6069 & 3 & 0.510 & 1800 \\
\hline LP Leo & $11: 36: 39.8$ & $-01: 25: 16$ & 16.5 & 0.5 & $V$ & 0.6492 & 3 & 0.682 & 2400 \\
\hline v370 Vir & $12: 06: 04.1$ & $-02: 12: 57$ & 14.7 & 0.9 & $V$ & 0.6992 & 3 & 0.455 & 1800 \\
\hline v408 Vir & $12: 40: 03.5$ & $-00: 04: 09$ & 16.5 & 0.7 & $V$ & 0.5858 & 3 & 0.391 & 3600 \\
\hline J1245-0419 & $12: 45: 03.0$ & $-04: 19: 11$ & 16.1 & 0.4 & $V$ & 0.7458 & 2 & 0.608 & 3600 \\
\hline ZZ Vir & $13: 23: 38.6$ & $-04: 21: 42$ & 13.7 & 1.2 & $B$ & 0.6841 & 1 & 0.614 & 600 \\
\hline WY Vir & $13: 35: 16.1$ & $-06: 58: 22$ & 13.0 & 1.2 & $B$ & 0.6093 & 1 & 0.769 & 300 \\
\hline
\end{tabular}

References. - (1) Samus et al. (2009); (2) Miceli et al. (2008); (3) Vivas et al. (2004).

layers of the star; variations of up to $70 \mathrm{~km} \mathrm{~s}^{-1}$ are found during a pulsation cycle (Smith 2004). Ideally, RR Lyrae stars should be observed when their phase is in the range $\phi=0.2-0.8$, corresponding to minimum light; maximum light occurs at $\phi=0$ and $\phi=1$.

All of the stars we observed have had their periods determined previously, allowing us to generate the accurate ephemerides required to determine the phases. All ephermerides were adjusted to agree with light curves derived from the data available in January 2013 from the Catalina Surveys Data Release 2 (CSDR2)1. The variability of RR Lyraes also constrains the length of the exposure times for the spectroscopic observations, as observing for too long could result in artificial broadening of the lines due to the changes in the atmospheric properties and radial velocities that are part of the pulsation cycle. For our targets, which are all RRab stars, we noted that the exposure times around any given phase should be less than 1.5 hours.

Observations of the first eight CEMP candidates were obtained in March 2013 using the Wide Field Spectrograph (WiFes; Dopita et al. 2007) on the ANU 2.3meter telescope at Siding Spring Observatory. The gratings used were both the B3000 and R7000, producing resolving powers of $R \sim 3000$ and 7000, respectively. The B3000 grating was deliberately selected because the wavelength coverage $(3500-5700 \AA)$ and resolution for this set up are directly compatible with the automated pipeline employed for atmospheric parameter determination, which is further described in Section 3. These data were reduced with the PyWiFeS software package (Childress et al. 2013).

The details of our observed RRab stars, including coordinates, maximum magnitude and amplitude for a given waveband, and the source of ephemeris for each star, are given in Table 1. We also list the exposure time and phase at mid-observation for each observed object.

\section{ATMOSPHERIC PARAMETERS}

The atmospheric parameters were determined with the n-SSPP, a version of the original SEGUE Stellar Parameter Pipeline (SSPP; Allende Prieto et al. 2008; Lee et al. 2008a, 2008b, 2011; Smolinski et al. 2011) that is capable of working with non-SEGUE spectroscopic data and without Sloan colors available. Given input moderateresolution spectra and color information for a number of photometric systems, the pipeline determines values for

\footnotetext{
${ }^{1}$ http://crts.caltech.edu/index.html
}

$T_{\text {eff }}, \log g$, and $[\mathrm{Fe} / \mathrm{H}]$ by averaging over a series of estimates for each parameter, based on spectral synthesis and $\chi^{2}$ minimization using grids of synthetic spectra, as well as other available techniques. In the case of v370 Vir, the $\log g$ was set at a value of 2.5 , as the estimates from the n-SSPP proved to be unreliable for this spectrum. We note that, for RR Lyrae stars in these observed phases, the value of $\log g$ should be less than 3.0 (Barcza \& Benk 2009). We adopt the nominal uncertainties of $150 \mathrm{~K}$ for $T_{\text {eff }}, 0.5$ dex for $\log g$, and 0.3 dex for $[\mathrm{Fe} / \mathrm{H}]$. For each RR Lyrae star, we set the microturbulent velocity at $\xi=3 \mathrm{~km} \mathrm{~s}^{-1}$, and adopt a conservative uncertainty of $1 \mathrm{~km} \mathrm{~s}^{-1}$.

Examples of the WiFeS moderate-resolution spectra for five of our program stars are shown in Figure [1, in order of decreasing metallicity. Note that one cannot directly compare the relative strengths of the $\mathrm{Ca}$ II $\mathrm{K}$ lines because of differences in the effective temperatures of these stars.

\section{CARBON-ABUNDANCE RATIOS}

Carbon-abundance ratios, $[\mathrm{C} / \mathrm{Fe}]$, are determined by spectral synthesis of the $\mathrm{CH} G$-band at $4300 \AA$. For this analysis, we employ the one-dimensional plane-parallel stellar atmospheres of Castelli \& Kurucz (2004), with no convective overshoot. Solar abundances used for reference are those of Asplund et al. (2009). The 2013 version of MOOG (Sneden 1973) was employed for spectral synthesis, and we minimized $\chi^{2}$ over the region $4290-4320$ $\AA$ for each spectrum.

Examples of this procedure can be seen in Figure 2. where we show the synthesis of the $\mathrm{CH} G$-band for four of our eight program stars. For each of the panels, the solid line denotes the best-fitting synthetic spectrum, corresponding to the best estimate of $[\mathrm{C} / \mathrm{Fe}]$ for each star. For reference, the dotted line denotes the solar value of the carbon-abundance rato, $[\mathrm{C} / \mathrm{Fe}]=0$. The atmospheric parameters and carbon abundances for all eight RR Lyrae stars in the sample are listed in Table 2. Figure 3 shows the $[\mathrm{C} / \mathrm{Fe}]$ ratios, as a function of $[\mathrm{Fe} / \mathrm{H}]$, for our program sample. There are seven stars which, at least within error bars, pass the criterion $[\mathrm{C} / \mathrm{Fe}] \geq+0.7$ for inclusion as CEMP stars.

We also include in Table 2 the low-resolution estimates of $[\mathrm{Fe} / \mathrm{H}]$ and $[\mathrm{C} / \mathrm{Fe}]$ from Christlieb et al. (2008), used for target selection, as well the $[\mathrm{Fe} / \mathrm{H}]$ estimates from other studies of RR Lyrae stars (Vivas et al. 2008; Layden 1995), available for three stars in our sample. 
Table 2

Atmospheric Parameters and C Abundances

\begin{tabular}{|c|c|c|c|c|c|c|c|c|}
\hline \multirow[b]{2}{*}{ Star } & \multirow[b]{2}{*}{$\Delta \log P^{a}$} & \multirow[b]{2}{*}{$T_{\text {eff }}(\mathrm{K})$} & \multicolumn{2}{|c|}{ This Study } & \multirow[b]{2}{*}[\mathrm{C}/\mathrm{Fe}]{} & \multicolumn{3}{|c|}{ Previous Estimates } \\
\hline & & & $\log g(\operatorname{cgs})$ & {$[\mathrm{Fe} / \mathrm{H}]$} & & $\begin{array}{c}\text { Chris } \\
{[\mathrm{Fe} / \mathrm{H}]}\end{array}$ & $\begin{array}{l}\text { lieb } \\
{[\mathrm{C} / \mathrm{Fe}]}\end{array}$ & $\begin{array}{l}\text { Other } \\
{[\mathrm{Fe} / \mathrm{H}]}\end{array}$ \\
\hline IV Leo & +0.0108 & 6609 & 2.82 & -1.48 & $+1.09(0.20)$ & -2.8 & +0.2 & \\
\hline LO Leo & +0.0606 & 6394 & 2.85 & -1.82 & $+0.73(0.25)$ & -2.7 & +1.1 & $-1.71^{\mathrm{c}}$ \\
\hline LP Leo & -0.0122 & 5882 & 2.61 & -1.64 & $+0.66(0.20)$ & -1.7 & -0.4 & $\ldots$ \\
\hline v370 Vir & +0.0833 & 6275 & 2.50 & -2.11 & $+1.36(0.20)$ & -2.8 & +1.7 & $-2.08^{\mathrm{c}}$ \\
\hline v408 Vir & -0.0177 & 6216 & 2.81 & -1.88 & $+0.62(0.30)$ & -1.4 & +0.7 & $\ldots$ \\
\hline J1245-0419 & +0.0535 & 5996 & 2.76 & -1.87 & $+0.34(0.30)$ & $\ldots$ & +2.4 & $\ldots$ \\
\hline ZZ Vir & +0.0736 & 6538 & 2.54 & -2.36 & $+1.71(0.25)$ & -2.9 & +0.9 & $\ldots$ \\
\hline WY Vir & +0.0603 & 6354 & 2.81 & -2.65 & $+1.35(0.30)$ & $\ldots$ & +3.9 & $-2.80^{\mathrm{d}}$ \\
\hline
\end{tabular}

a Shift in log period from the locus of Oo I stars in Bailey diagram.

b Low-resolution estimates of metallicities and carbon-abundance ratios from Christlieb et al. (2008).

c Vivas et al. (2008).

d Layden 1995.

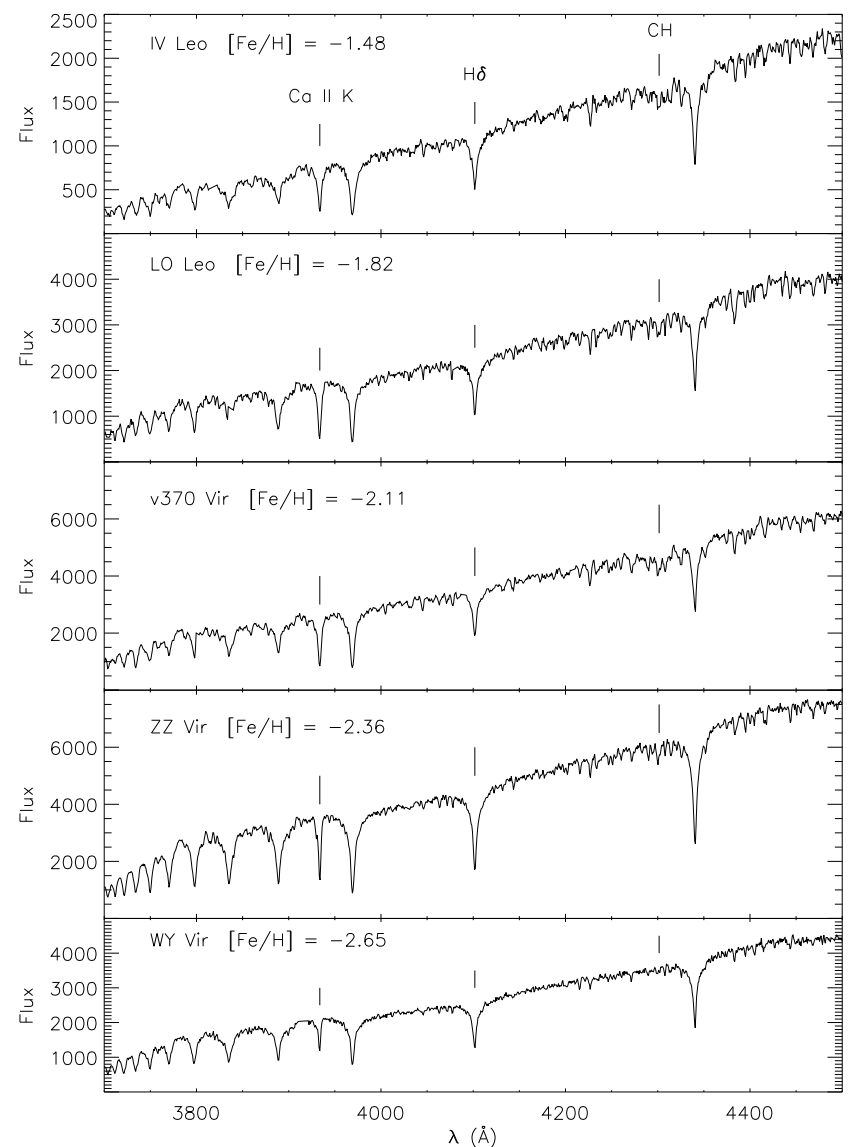

Figure 1. Moderate-resolution WiFeS spectra for five program stars in order of decreasing metallicity. The Ca II K line, the $\mathrm{H} \delta$ Balmer line, and the $\mathrm{CH} G$-band for each star are indicated by the vertical lines.

Because the Christlieb et al. (2008) calculation of $[\mathrm{C} / \mathrm{Fe}]$ is based on the $\mathrm{CH} G$-band strength at a given color, it does not require an estimate of $[\mathrm{Fe} / \mathrm{H}]$. If the $\mathrm{Ca}$ II $\mathrm{K}$ line was too weak (on the prism spectrum) to be detected, an estimate of $[\mathrm{Fe} / \mathrm{H}]$ could not be obtained. Even with the aforementioned large errors on $[\mathrm{Fe} / \mathrm{H}]$ and $[\mathrm{C} / \mathrm{Fe}]$ from the low-resolution prism spectra, we note that five out of six stars with estimates of $[\mathrm{C} / \mathrm{Fe}] \geq+0.7$ from Christlieb et al. (2008) turned out to be CEMP stars.
Furthermore, the additional estimates of metallicity for LO Leo, v370 Vir, and WY Vir agree with our $[\mathrm{Fe} / \mathrm{H}]$ estimates to within 0.15 dex.

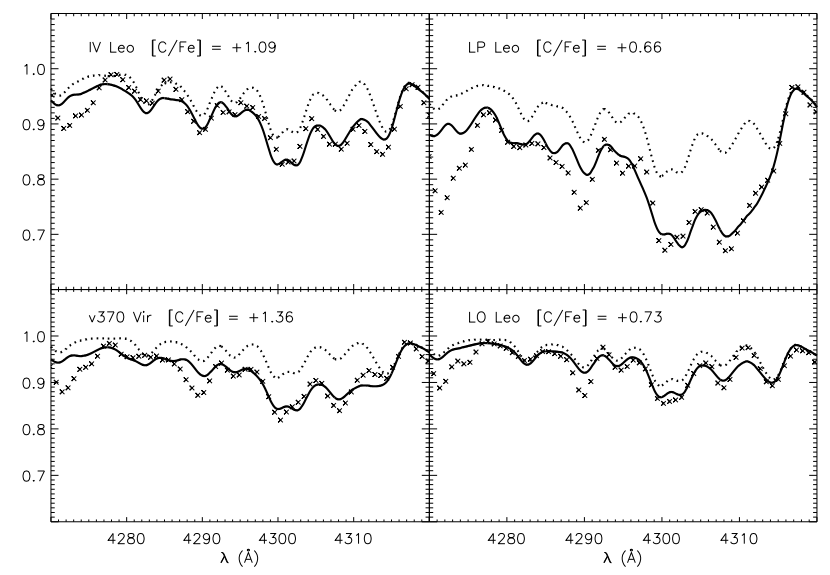

Figure 2. Synthesis of the $\mathrm{CH} G$-band for four stars in the sample. Crosses represent the observed spectrum for each object. The solid lines are the best-fitting synthetic spectra, while the dotted lines show the solar value, $[\mathrm{C} / \mathrm{Fe}]=0$.

\subsection{Uncertainties}

Uncertainties for the carbon-abundance ratios, $\delta[\mathrm{C} / \mathrm{Fe}]$, were determined directly from the spectral synthesis. We estimated the upper and lower bounds by demanding that their associated synthetic spectra completely enclose the $\mathrm{CH} G$-band feature from which we estimate $[\mathrm{C} / \mathrm{Fe}]$. An example of this technique is shown in Figure 4. The uncertainty in $[\mathrm{C} / \mathrm{Fe}]$ for each star is shown in parentheses next to its carbon-abundance ratio in Table 2. The error on $[\mathrm{C} / \mathrm{Fe}]$ for this sample ranges from $0.2-0.3$ dex.

We also have estimated the systematic uncertainties, those due to adopted errors on our atmospheric parameters, for each star in the sample. The average values of these systematic uncertainties are listed in Table 3. It is clear that the two largest possible systematic uncertainties arise from errors associated with effective temperature and metallicity. Even very conservative adopted 


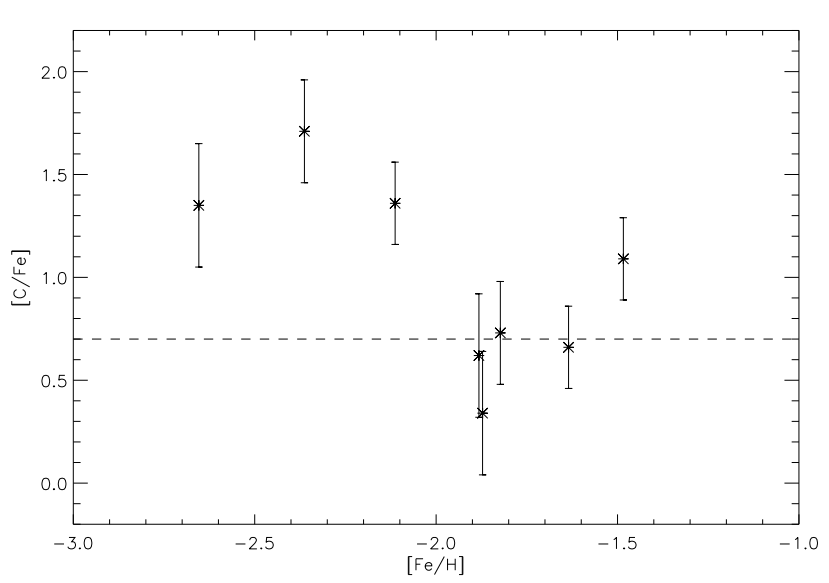

Figure 3. $[\mathrm{C} / \mathrm{Fe}]$ vs. $[\mathrm{Fe} / \mathrm{H}]$ for the entire program sample. Stars with $[\mathrm{C} / \mathrm{Fe}]$ values falling above the dashed line at $[\mathrm{C} / \mathrm{Fe}]=+0.7$ are classified as CEMP stars. There are two stars that lie very close to the line, while another is consistent with it to within the error bars. We consider all but one of these stars to be CEMP stars.

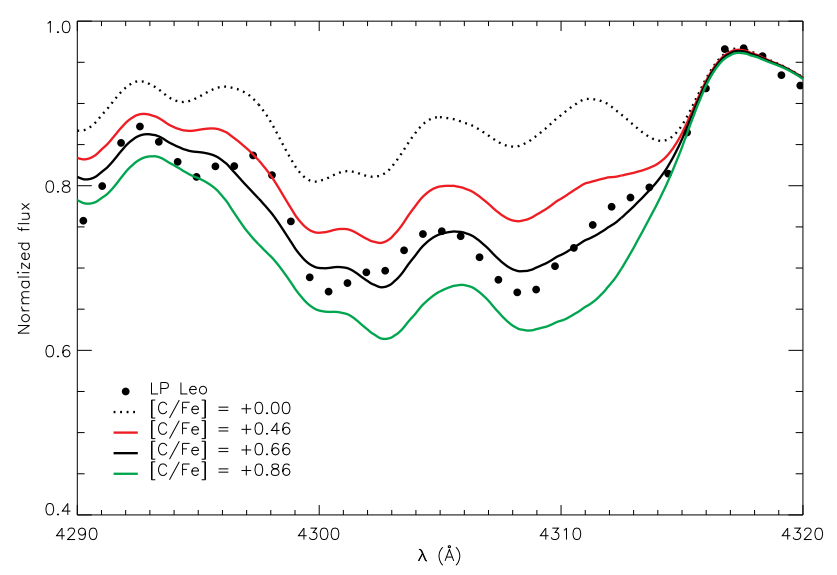

Figure 4. Example of $[\mathrm{C} / \mathrm{Fe}]$ uncertainty determination. The solid line represents the best-fitting spectrum, while the red and green lines represent the synthetic spectra associated with our estimated error of $\delta[\mathrm{C} / \mathrm{Fe}]=0.2$ dex for LP Leo. Again, the dotted line show the solar value, $[\mathrm{C} / \mathrm{Fe}]=0$.

Table 3

Systematic $[\mathrm{C} / \mathrm{Fe}]$ Uncertainties

\begin{tabular}{lc}
\hline \hline & $\Delta[\mathrm{C} / \mathrm{Fe}]$ \\
\hline$T_{\text {eff }}-150 \mathrm{~K}$ & -0.30 \\
$T_{\text {eff }}+150 \mathrm{~K}$ & +0.28 \\
$\log g-0.5 \mathrm{dex}$ & +0.16 \\
$\log g+0.5 \mathrm{dex}$ & -0.16 \\
{$[\mathrm{Fe} / \mathrm{H}]-0.3 \mathrm{dex}$} & +0.36 \\
{$[\mathrm{Fe} / \mathrm{H}]+0.3 \mathrm{dex}$} & -0.43 \\
$\xi-1 \mathrm{~km} \mathrm{~s}^{-1}$ & +0.05 \\
$\xi+1 \mathrm{~km} \mathrm{~s}^{-1}$ & -0.06
\end{tabular}

uncertainties for surface gravity and microturbulent velocity do not result in the propagation of significant errors on our estimated $[\mathrm{C} / \mathrm{Fe}]$ abundances.

\section{OOSTERHOFF CLASSIFICATION}

Milky Way globular clusters can be divided into two groups, known as Oosterhoff groups, based on the prop- erties of their RR Lyrae stars, with Oosterhoff II (Oo II) clusters being more metal poor and having longer average RR Lyrae periods than Oosterhoff I (Oo I) clusters (Oosterhoff 1939). Like the RR Lyrae variables studied in globular clusters, RR Lyrae field stars have been shown to exhibit properties of both Oo I and Oo II systems (Kinemuchi et al. 2006), although the separation between the two populations is far less distinct. Following Kinman et al. (2012), we study the Oosterhoff types of the CEMP RR Lyrae field stars in order to compare to classical Oosterhoff systems, as well as to explore how the presence of carbon in the atmosphere might affect the nature of the pulsation.

The Oosterhoff types of the observed CEMP RR Lyrae stars are determined based on their location in the period-amplitude plot (or Bailey diagram), as shown in the top panel of Figure 5. The red and blue dashed lines show the typical loci for Oo I and Oo II RRab stars (Cacciari et al. 2005). More quantitatively, stars with $\Delta \log P>0.50$ are classified as Oosterhoff II, where $\Delta \log P$ is the shift in the log period from the locus of Oo I stars at a given amplitude. The values of $\Delta \log P$ for each object as given in the second column of Table 2, Based on the periods and $V$-band amplitudes of the sample, we classify three stars as Oo I types and the remaining five as Oo II types (solid circles). Also shown in this figure are the periods and amplitudes for a sample of RR Lyrae stars with $[\mathrm{Fe} / \mathrm{H}] \leq-1.0$ from Wallerstein et al. (2009) (open circles). Unlike our sample, these metal-poor RR Lyrae stars do not exhibit carbon enhancements. In fact, the majority of them have carbon-abundance ratios that are significantly sub-solar $([\mathrm{C} / \mathrm{Fe}] \sim-0.5)$. These lower carbon abundances can perhaps be attributed to $\mathrm{CN}$ cycling having occurred as these stars ascended the giant branch. In this case, the stars would likely have originally been carbon-normal metal-poor stars, with $[\mathrm{C} / \mathrm{Fe}]$ $\sim+0.0$, prior to having undergone evolutionary mixing effects (see, e.g., Figure 3 in Lucatello et al. 2006).

In the lower panel of Figure 5, we compare the metallicities and periods of the Oo I and Oo II RR Lyrae stars from above to the mean values found in globular clusters (shown as crosses), taken from Bono et al. (2007). These globular cluster classifications are based on an average of many RRab stars within each cluster, and there is an appreciable range in pulsation periods among the individual objects. Thus, a direct comparison of field RR Lyrae stars to mean values for globular clusters is perhaps not meaningful. Nevertheless, we recognize that our observed Oo I and Oo II field stars are consistent with the general behavior of Oo I and Oo II clusters.

Given that there are very few RR Lyrae stars with available carbon abundances, it is difficult to fully assess how the presence of enhanced carbon in the atmosphere might affect the physical nature of these stars. The carbon-weak field RR Lyrae stars from Wallerstein et al. (2009) appear to exhibit longer pulsation periods than their carbon-enhanced counterparts, even though the metallicities of the two samples are very similar. However, the periods of the carbon-weak stars are still within the range of those periods associated with Oo II stars, and their separation from the Oo II locus is within the level of scatter that is seen in Oo II clusters. We therefore are unable to conclude that the presence of enhanced carbon influences the dynamics of pulsation. Nevertheless, 
we seek to obtain carbon-abundance information for a much larger sample of RR Lyrae stars, since the contrast in the behavior of pulsation between carbon-enhanced and carbon-weak samples could be indicative that the presence (or lack) of significant carbon has a physical effect on the dynamics of pulsation or other properties of horizontal-branch stars.

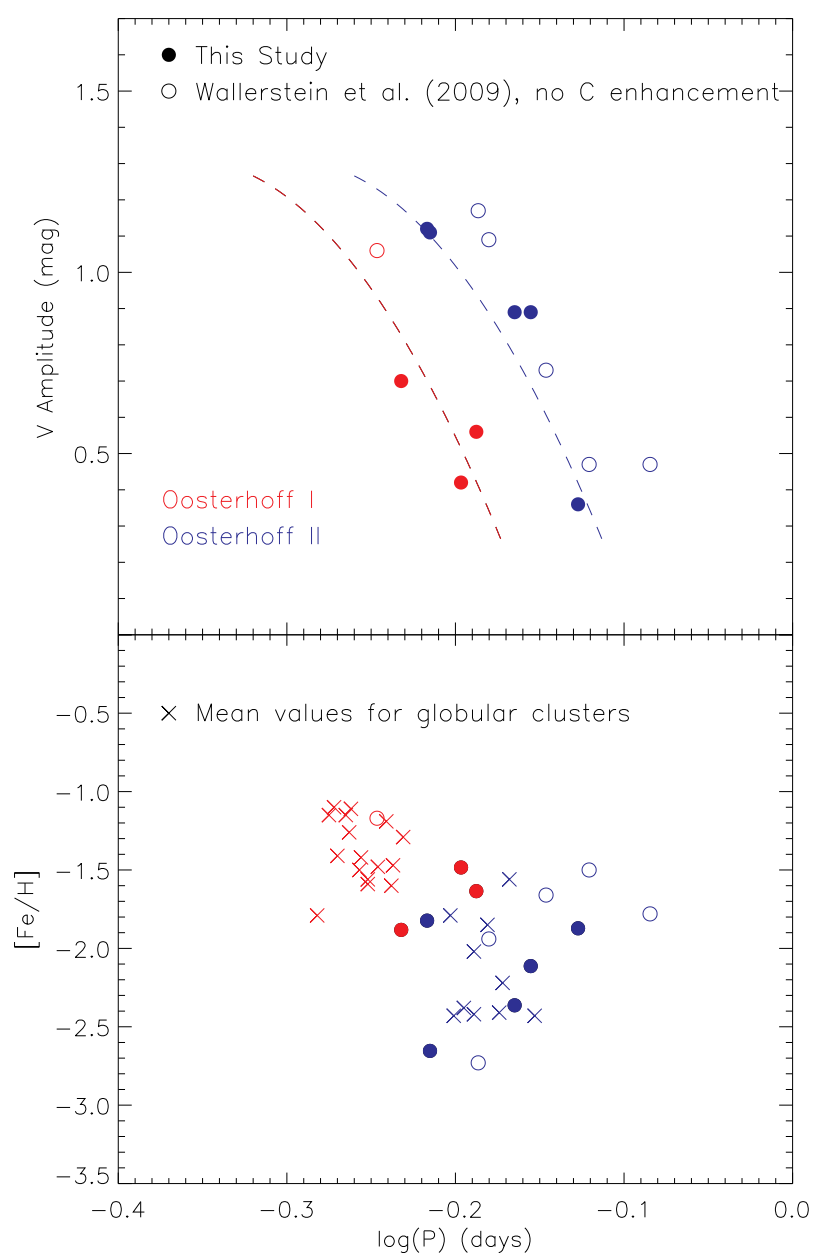

Figure 5. Oosterhoff classification of CEMP RR Lyrae stars. Top panel: Period-amplitude diagram for program stars (filled circles) and those from Wallerstein et al. (2009) (open circles). Oo I (red) and Oo II (blue) types are assigned based on their proximity to the trends of Cacciari et al. (2005) (dashed lines). Bottom panel: Metallicity vs. period for RR Lyrae stars (same symbols as above) compared to the mean values found in studies of globular clusters (Bono et al. 2007).

\section{THEORETICAL MODELS OF CEMP RR LYRAE STARS}

Recently, Stancliffe et al. (2013) conducted thorough simulations of the binary progenitor systems of CEMP RR Lyrae stars with $[\mathrm{Fe} / \mathrm{H}]=-2.3$. In these models, a range of masses of accreted material from AGB donor stars (with initial masses of $1-2 \mathrm{M}_{\odot}$ ) are simulated, following the prescription of Stancliffe (2009). Post accretion, the secondary star is then evolved all the way through full giant-branch evolution to the horizontal-branch stage. Using the detailed yields for low-metallicity AGB stars, computed by Lugaro et al. (2012), the composition of the companion as it evolves is
Table 4

Theoretical $[\mathrm{C} / \mathrm{Fe}]$ from Stancliffe et al. (2013)

\begin{tabular}{ccccc}
\hline \hline AGB Donor Mass & Accreted Mass & \multicolumn{3}{c}{$[\mathrm{C} / \mathrm{Fe}]$} \\
\cline { 3 - 5 }$\left(\mathrm{M}_{\odot}\right)$ & $\left(\mathrm{M}_{\odot}\right)$ & $\mathrm{C}$ & $\mathrm{C} / \mathrm{TH}$ & $\mathrm{C} / \mathrm{TH} / \mathrm{GS}$ \\
\hline 1.0 & 0.1 & +0.72 & +0.65 & +0.52 \\
1.0 & 0.01 & -0.28 & -0.23 & -0.62 \\
1.0 & 0.001 & -0.55 & -0.52 & -0.93 \\
& & & & \\
1.5 & 0.1 & +2.26 & +2.07 & +2.07 \\
1.5 & 0.05 & +1.73 & $\ldots$ & $\ldots$ \\
1.5 & 0.02 & +0.97 & $\ldots$ & $\ldots$ \\
1.5 & 0.01 & +0.52 & +0.48 & +0.39 \\
& 0.001 & -0.58 & -0.44 & -0.57 \\
2.0 & & & & \\
2.0 & 0.1 & +2.58 & +2.02 & +2.04 \\
& 0.01 & +0.95 & +0.62 & +0.61 \\
& 0.001 & -0.52 & -0.36 & -0.42 \\
\hline
\end{tabular}

predicted, for any species from $\mathrm{H}$ to $\mathrm{Pb}$ (the terminus of the $s$-process), rather than just for the light elements (as computed by Stancliffe 2009). We refer the interested reader to Stancliffe et al. (2013) for details of the code. Throughout these simulations, various evolutionary mixing scenarios are considered, including combinations of standard convection, thermohaline mixing, and gravitational settling. The final theoretical abundances of the CEMP RR Lyrae stars are thus the result of complete consideration of primary mass, AGB nucleosynthesis, accretion mass, and subsequent mixing physics (both convective and non-convective) for a particular set of initial conditions. Therefore, these models present themselves as realistic comparisons to our observed CEMP RR Lyrae stars.

While the theoretical models include abundance information for several elements, including those formed via the $s$-process, the sample of observed CEMP RR Lyrae stars presently only have carbon-abundance information, and therefore, we restrict our theoretical comparisons to carbon alone. For reference, a table of the theoretical $[\mathrm{C} / \mathrm{Fe}]$ abundances used for our comparison is given in Table 4. For each combination of donor mass and accreted mass, the resultant $[\mathrm{C} / \mathrm{Fe}]$ values are given under three different assumptions of mixing physics: standard convection only $(\mathrm{C})$, standard convection and thermohaline mixing $(\mathrm{C} / \mathrm{TH})$, and standard convection, thermohaline mixing, and gravitational settling (C/TH/GS).

\section{DISCUSSION}

In comparing our $[\mathrm{C} / \mathrm{Fe}]$ abundances to the theoretical abundances of Stancliffe et al. (2013), we explore the likely AGB donor masses, accretion masses, and mixing histories associated with the progenitor systems of our observed CEMP RR Lyrae stars. In Figure 6, the dependence of $[\mathrm{C} / \mathrm{Fe}]$ on donor mass, accreted mass, and mixing mechanism is shown for some of the theoretical scenarios given in Table 4 . It is clear from the top panel of Figure 6] that the $[\mathrm{C} / \mathrm{Fe}]$ abundances are strongly dependent on the assumed accreted mass, while the abundances are much less sensitive to the assumed mixing mechanism (bottom panel). Indeed, given that the errors on our $[\mathrm{C} / \mathrm{Fe}]$ estimates are around $\sim 0.25$ dex, we are unable to place any conclusive constraints on the evolutionary mixing processes that have occurred in the ob- 
served stars. Nevertheless, the AGB donor mass and the mass of the accreted material are constrained for the entire sample.
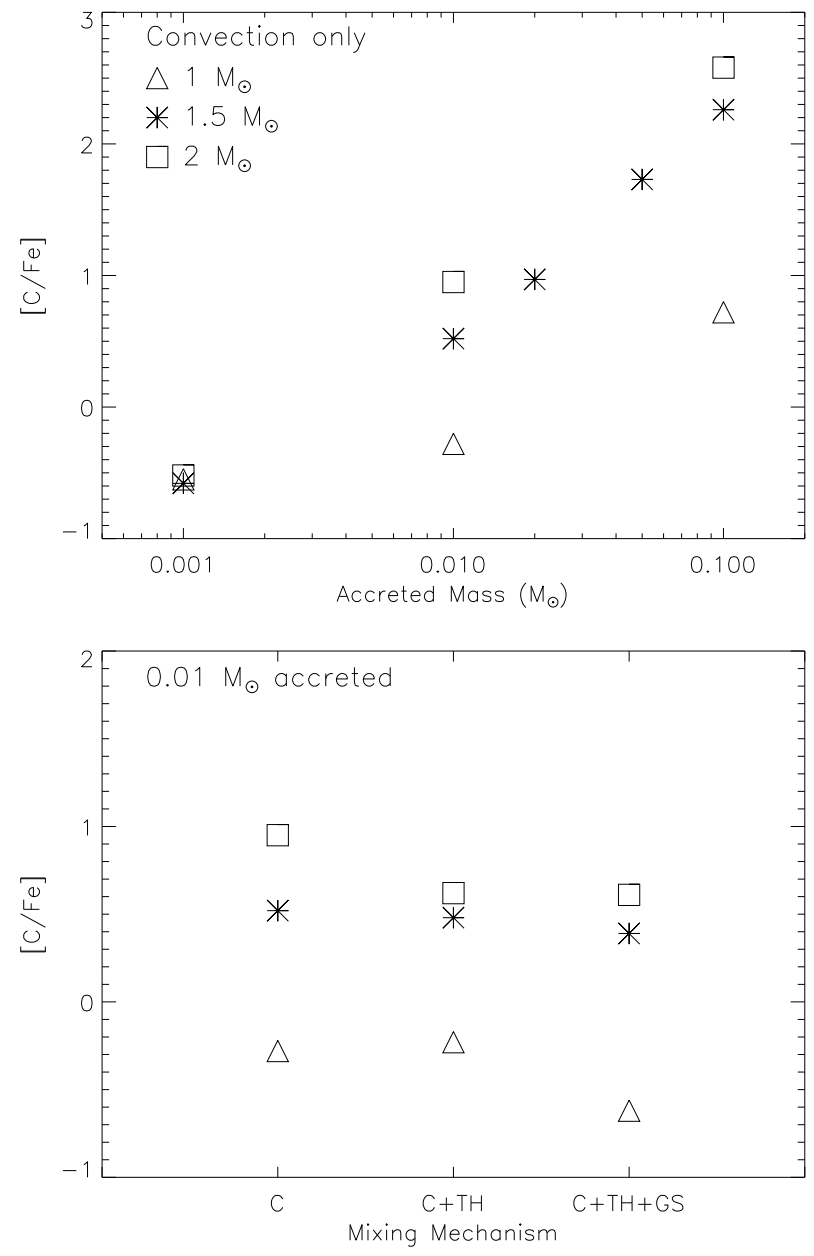

Figure 6. Theoretical $[\mathrm{C} / \mathrm{Fe}]$ from Stancliffe et al. (2013). Top panel: Dependence of $[\mathrm{C} / \mathrm{Fe}]$ on accreted mass for a range of $\mathrm{AGB}$ donor masses. Only the standard convection scenario is shown. Bottom panel: Dependence of $[\mathrm{C} / \mathrm{Fe}]$ on mixing scenario for a range of AGB donor masses (same symbols as above) for an accretion mass of $0.01 \mathrm{M}_{\odot}$.

\section{1. $[\mathrm{Fe} / \mathrm{H}]$ Considerations and $[\mathrm{C} / \mathrm{Fe}]$ Corrections}

All of the theoretical models available for comparison (Stancliffe et al. 2013) correspond to $[\mathrm{Fe} / \mathrm{H}]=-2.3$, and include as inputs AGB yields for $[\mathrm{Fe} / \mathrm{H}]=-2.3$ (Lugaro et al. 2012). However, the metallicities of the observed CEMP RR Lyrae stars, given in Table 2, span a wide range $(-2.65<[\mathrm{Fe} / \mathrm{H}]<-1.48)$. Given that the theoretical carbon yields of metal-poor AGB stars vary dramatically for models of different metallicity (for reference, see Figure 5 from Campbell \& Lattanzio 2008), it is necessary to apply a metallicity-dependent correction to the theoretical $[\mathrm{C} / \mathrm{Fe}]$ abundances prior to comparison to our observed $[\mathrm{C} / \mathrm{Fe}]$.

To determine the metallicity-dependent $[\mathrm{C} / \mathrm{Fe}]$ corrections, we employed new theoretical AGB yields for $[\mathrm{Fe} / \mathrm{H}]=-1.4$ and -1.8 (Karakas et al. 2014), in combination with the $[\mathrm{Fe} / \mathrm{H}]=-2.3$ yields, for an $\mathrm{AGB}$ donor mass of $1.7 \mathrm{M}_{\odot}$. With the $[\mathrm{C} / \mathrm{Fe}]$ values for these three different metallicities in hand, we performed a linear interpolation with $[\mathrm{Fe} / \mathrm{H}]$ in order to estimate the $[\mathrm{C} / \mathrm{Fe}]$ correction, $\Delta[\mathrm{C} / \mathrm{Fe}]$, for each observed RR Lyrae star. These eight individual corrections were then applied to the theoretical $[\mathrm{C} / \mathrm{Fe}]$ abundances in Table 4 , prior to comparison with our results. While the corrections were estimated from the direct AGB yields instead of the surface abundances of the evolved secondary, we note that the subsequent relative dilution of the material, once accreted, should not vary appreciably with metallicity. A test of these adopted corrections has been carried out for one scenario $\left(2 \mathrm{M}_{\odot}\right.$ donor, $0.1 \mathrm{M}_{\odot}$ accretion, $[\mathrm{Fe} / \mathrm{H}]=-1.8)$, and the final, post-evolution $[\mathrm{C} / \mathrm{Fe}]$ is consistent with our adopted $[\mathrm{C} / \mathrm{Fe}]$ based on the correction described above.

For the interpretation of these results, we apply the aforementioned $[\mathrm{C} / \mathrm{Fe}]$ corrections directly to the models, in order to approximate the theoretical yields for RR Lyrae stars of different metallicities. However, for visual simplicity, we show graphically in Figure 7 the opposite shifts applied to the $[\mathrm{C} / \mathrm{Fe}]$ estimates of the individual $\mathrm{RR}$ Lyrae stars. In this figure, the open squares are the measured $[\mathrm{C} / \mathrm{Fe}]$ values, and the red squares show the $[\mathrm{C} / \mathrm{Fe}]$ values after they have been appropriately scaled. The horizontal lines show the theoretical $[\mathrm{C} / \mathrm{Fe}]$ values for different model scenarios, for $[\mathrm{Fe} / \mathrm{H}]=-2.3$. Hence, for theoretical comparison, one should focus only on the shifted values of [C/Fe] (shown as red squares) in Figure 7 as they have been shifted in order to match the metallicity of the models, $[\mathrm{Fe} / \mathrm{H}]=-2.3$.

\subsection{Progenitor Scenarios for Individual CEMP RR Lyrae Stars}

LP Leo, v370 Vir, LO Leo, ZZ Vir, v408 Vir - The $[\mathrm{C} / \mathrm{Fe}]$ abundances of these five CEMP RR Lyrae stars, after the applied correction on $[\mathrm{C} / \mathrm{Fe}]$ is considered, are indicative of progenitor systems that share several common properties. In Figure 7, it is evident that none of these five stars are consistent with the lowest-mass AGB donor, $1 \mathrm{M}_{\odot}$, even when the highest accreted mass is considered. For lower accretion masses, the predicted values of $[\mathrm{C} / \mathrm{Fe}]$ are sub-solar and not shown in the figure. In fact, for all AGB donor masses considered, the lowest accretion masses $\left(0.001 \mathrm{M}_{\odot}\right)$ are inconsistent with this sample, as the resultant $[\mathrm{C} / \mathrm{Fe}]$ abundances are all sub-solar. Furthermore, these observed abundances are inconsistent with large accretion masses, $0.1 \mathrm{M}_{\odot}$, regardless of the AGB donor mass. For these five stars, we therefore can reasonably constrain the AGB donor mass as being $>1 \mathrm{M}_{\odot}$ and the accreted mass as being $<0.1$ $\mathrm{M}_{\odot}$. Indeed, these five CEMP RR Lyrae stars are consistent with AGB donors in the range $\sim 1.5-2.0 \mathrm{M}_{\odot}$ and appear to have accreted a few hundredths of a solar mass of AGB material prior to their subsequent evolution.

$W Y$ Vir - The lowest-metallicity star in the sample, WY Vir, has a carbon-abundance ratio that exactly matches two of the theoretical scenarios, both $0.02 \mathrm{M}_{\odot}$ of material accreted from a $1.5 \mathrm{M}_{\odot}$ donor and $0.01 \mathrm{M}_{\odot}$ of material accreted from a $2.0 \mathrm{M}_{\odot}$ donor, as shown in Figure 77. As such, it appears to be indicative of the same type of progenitor system described for the five stars above. However, given the large uncertainty associated with the measured [C/Fe] (0.3 dex), there remains 


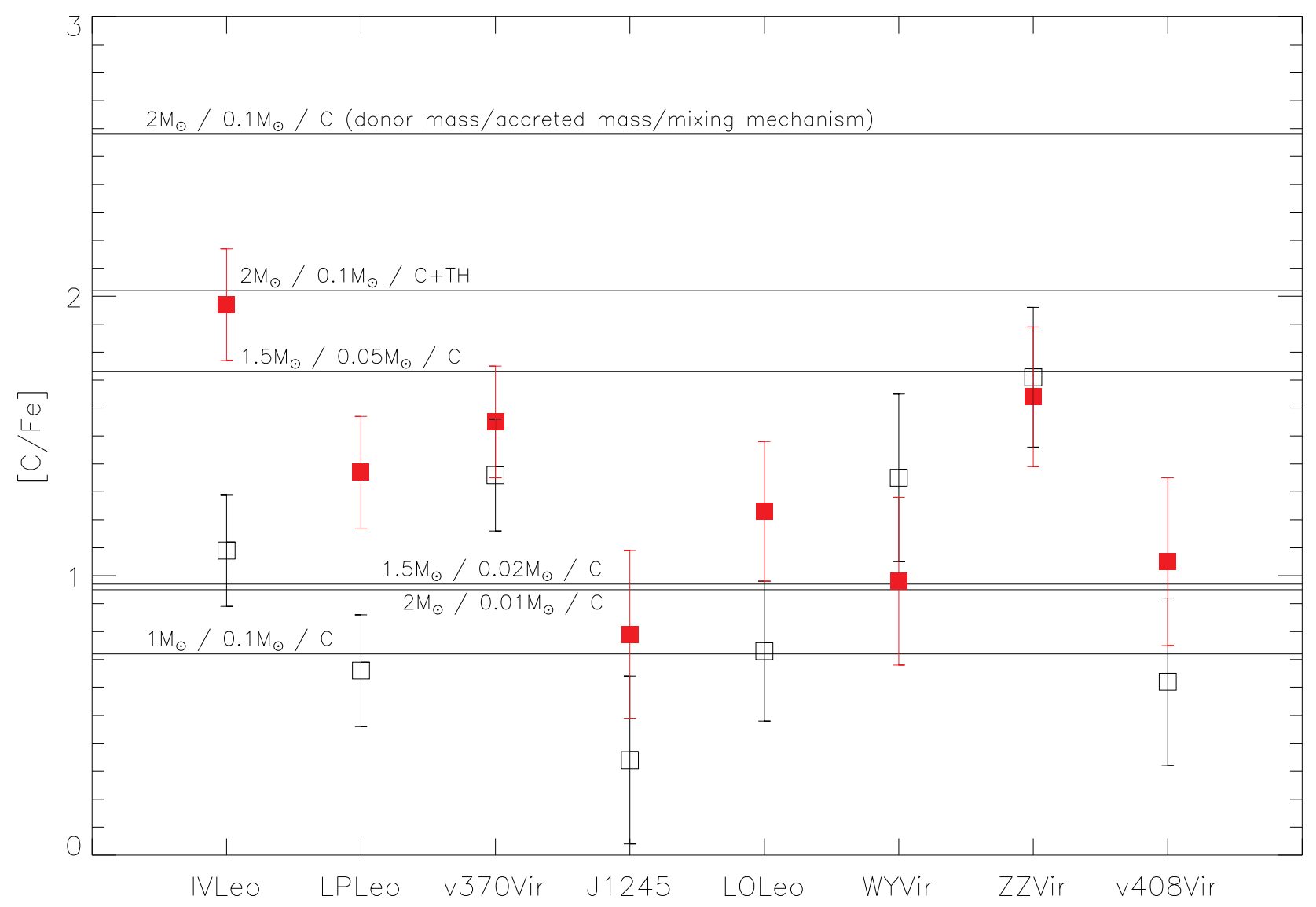

Figure 7. Comparison of $[\mathrm{C} / \mathrm{Fe}]$ abundances in CEMP RR Lyrae stars to theoretical models (horizontal lines). The black open squares are the measured abundances. The solid red squares have been shifted to match the metallicity of the models, and therefore they are the focus for accurate comparison. See text for details.

a possibility that $\sim 0.1$ solar masses of material could have been accreted from a low-mass AGB companion of $\sim 1$ solar mass. While this ambiguity exists, it is nevertheless evident that such large accretion masses cannot have come from the larger-mass donors considered. And, as for the rest of the sample, we can rule out all scenarios of very low accretion masses of $0.001 \mathrm{M}_{\odot}$.

IV Leo - The highest-metallicity star in the sample, IV Leo, presents the only opportunity to explore the different evolutionary mixing mechanisms explored in these models. Similar to all of the stars described above, its measured $[\mathrm{C} / \mathrm{Fe}]$ agrees with models of accretion of a few hundredths of a solar mass from a 1.5 or 2 solar mass AGB donor. Interestingly, another possible progenitor scenario includes the accretion of a large amount of mass $\left(\sim 0.1 \mathrm{M}_{\odot}\right)$ from the 1.5 or 2 solar mass donor, but only if one considers that thermohaline mixing, or thermohaline mixing and gravitational settling, was/were active during a previous evolutionary phase. Without these extra mixing processes, such a large accretion mass would result in a much larger $[\mathrm{C} / \mathrm{Fe}]$ than we measure in this star. It is possible, therefore, to consider that a very large amount of mass was accreted under these certain mixing physics constraints. We confidently rule out any scenario with an AGB companion of 1 solar mass, as well as all cases of 0.001 solar mass accretion.
J1245-0419 - The only star in this sample which does not formally meet the criteria for CEMP stars, J1245-0419, has the most ambiguous carbon-abundance ratio when it comes to theoretical comparison, as can be seen in Figure 7 . The value of $[\mathrm{C} / \mathrm{Fe}]$ for this star is fully consistent with a wide range of scenarios, including all three donor masses. If the AGB companion was only 1 $\mathrm{M}_{\odot}$, then accretion must have been very high. For the 1.5 and $2 \mathrm{M}_{\odot}$ cases, accretion mass would have to be around 0.01 or 0.02 solar masses. Regardless of this ambiguity, we can with certainty rule out a large accretion mass from a 1.5 or 2 solar mass donor.

\section{CONCLUSIONS}

We have obtained moderate-resolution spectroscopy and estimated stellar parameters and carbon-abundance ratios, $[\mathrm{C} / \mathrm{Fe}]$, for a sample of $8 \mathrm{RR}$ Lyrae stars, resulting in the identification of seven new CEMP RR Lyrae variables. As there were previously only two such stars discovered, we significantly increase the sample size of these objects.

Five of the stars are Oosterhoff II type variables and the remaining three are Oosterhoff I, based on their periods, $V$ amplitudes, and metallicities. Upon comparison to a sample of metal-poor RR Lyrae stars with subsolar $[\mathrm{C} / \mathrm{Fe}]$, we find a separation between pulsation period, suggesting that the presence (or lack) of sufficient 
carbon could have a physical effect on the dynamics of variability. However, larger sample sizes of both carbonenhanced and carbon-weak RR Lyrae stars remain necessary to fully explore this claim.

We compared the measured $[\mathrm{C} / \mathrm{Fe}]$ abundances in the CEMP RR Lyrae stars to new theoretical models of AGB binary mass transfer and subsequent evolution. We find that the majority of the are consistent with AGB donor masses of $\sim 1.5-2.0 \mathrm{M}_{\odot}$ and accretion masses of $\sim$ $0.01-0.05 \mathrm{M}_{\odot}$. Large accretion masses, on the order of $0.1 \mathrm{M}_{\odot}$ remain possible for a few objects. All eight stars are inconsistent with scenarios in which very low accretion masses of $\sim 0.001 \mathrm{M}_{\odot}$ are considered, which is to be expected, given the small contribution of carbon these donors provide and the large degree of dilution that this material undergoes.

Future studies should include high-resolution spectroscopy of CEMP RR Lyrae stars, as well as other nonvariable horizontal-branch stars, in order to explore more elements for theoretical comparison, in particular those associated with the $s$-process. With a larger set of highprecision abundance estimates, we can delve more deeply into the likely mixing history of these stars (by both convective and non-convective processes). Furthermore, we plan to expand the set of theoretical models to include those of different metallicities for a more complete set of donor masses and accretion masses.

CRK acknowledges support from the Australian Research Council (Super Science Fellowship; FS110200016). RJS is the recipient of a Sofja Kovalevskaja Award from the Alexander von Humboldt Foundation. TCB acknowledges partial support from grant PHY 08-22648; Physics Frontier Center/Joint Institute for Nuclear Astrophysics (JINA), awarded by the US National Science Foundation. The authors thank Amanda Karakas for providing AGB yields for different metallicities. VMP acknowledges support from the Gemini Observatory. SR acknowledges FAPESP. HR acknowledges CNPq, CAPES and PROEX.

\section{REFERENCES}

Abate, C., Pols, O. R., Izzard, R. G., Mohamed, S. S., \& de Mink, S. E. 2013, A\&A, 552, A26

Allende Prieto, C., Sivarani, T., Beers, T. C., et al. 2008, AJ, 136, 2070

Aoki, W., Beers, T. C., Christlieb, N., Norris, J. E., Ryan, S. G., \& Tsangarides, S. 2007, ApJ, 655, 492

Asplund, M., Grevesse, N., Sauval, A. J., \& Scott, P. 2009, ARA\&A, 47, 481

Barcza, S. \& Benk, J. M. 2009, AIP Conf. Proc., 1170, 250

Beers, T. C., Preston, G. W., \& Shectman, S. A. 1992, AJ, 103, 1987

Beers, T. C., \& Christlieb, N. 2005, ARA\&A, 43, 531

Bono, G., Caputo, F., \& Criscienzo, M. 2007, A\&A, 476, 779

Cacciari, C., Corwin, T., \& Carney, B. 2005, AJ, 129, 267

Campbell, S. W., \& Lattanzio, J. C. 2008, A\&A, 490, 769

Carollo, D., Beers, T. C., Bovy, J., et al. 2012, ApJ, 744, 195

Castelli, F., \& Kurucz, R. L. 2004, arXiv:astro-ph/0405087

Childress, M. J., Vogt, F. P. A., Nielsen, J., \& Sharp, R. G. 2013, arXiv:1311.2666 1

Christlieb, N., Schörck, T., Frebel, A., Beers, T. C., Wisotzki, L., \& Reimers, D. 2008, A\&A, 484, 721
Cohen, J. G., Shectman, S., Thompson, I., et al. 2005, ApJ, 633, 109

Dopita, M., Hart, J., McGregor, P., Oates, P., Bloxham, G., \& Jones, D. 2007, Ap\&SS, 310, 255

Frebel, A., Christlieb, N., Norris, J. E., et al. 2006, ApJ, 652, 1585

Hansen, C. J., Nordström, B., Bonifacio, P., et al. 2011, A\&A, 527,65

Hansen, T., Andersen, J., Nordström, B., Buchhave, L. A., \& Beers, T. C. 2011, ApJ, 743, 1

Hansen, T., Anderson, J., \& Nordström, B. 2013, Proceedings of the XII International Symposium on Nuclei in the Cosmos (NIC XII), Proceedings of Science, 146, 193

Herwig, F. 2005, ARA\&A, 43, 435

Hirschi, R., Fröhlich, C., Liebendörfer, M., \& Thieleman, F.-K. 2006, Reviews of Modern Astronomy, 19, 101

Ito, H., Aoki, W., Beers, T. C., Tominaga, N., Honda, S., \& Carollo, D. 2013, ApJ, 733, 33

Karakas, A. I., Marino, A. F., \& Nataf, D. M. 2014, ApJ, 784, 32

Kinemuchi, K., Smith, H. A., Wozniak, P. R., \& McKay, T. A. 2006, AJ, 132, 1202

Kinman, T. D., Aoki, W., Beers, T. C., \& Brown, W. R. 2012, ApJ, 755, L18

Kobayashi, C., Tominaga, N., \& Nomoto, K. 2011, ApJ, 730, L14 Layden, A. 1995, AJ, 110, 2288

Lee, Y. S., Beers, T. C., Sivarani, T., et al. 2008a, AJ, 136, 2022

Lee, Y. S., Beers, T. C., Sivarani, T., et al. 2008b, AJ, 136, 2050

Lee, Y. S., Beers, T. C., Allende Prieto, C., et al. 2011, AJ, 141, 90

Lee, Y. S., Beers, T. C., Masseron, T., et al. 2013, AJ, 146, 132

Lucatello, S., Beers, T. C., Christlieb, N., et al. 2006, ApJ, 652, 37

Lugaro, M., Karakas, A. I., Stancliffe, R. J., \& Rijs, C. 2012, ApJ, 747, 2

Marsteller, B., Beers, T. C., Rossi, S., Christlieb, N., Bessell, M., \& Rhee, J. 2005, Nucl. Phys. A, 758, 312

Masseron, T., Johnson, J. A., Lucatello, S., et al. 2012, ApJ, 751, 14

Meynet, G., Ekström, S., \& Maeder, A. 2006, A\&A, 447, 623

Meynet, G.,Hirschi, R., Ekström, S., et al. 2010, A\&A, 521, 30

Miceli, A., Rest, A., Stubbs, C., et al. 2008, ApJ, 678, 865

Nomoto, K., Kobayashi, C., \& Tominaga, N. 2013, ARA\&A, 51, 457

Norris, J. E., Ryan, S. G., \& Beers, T. C. 1997, ApJ, 488, 350

Norris, J. E., Christlieb, N., Korn, A. J., et al. 2007, ApJ, 670, 774

Norris, J. E., Yong, D., Bessell, M. S., et al. 2013, ApJ, 762, 28

Oosterhoff, P. T. 1939, The Observatory, 62, 104

Preston, G. W., Thompson, I. A., Sneden, C., Stachoqski, G., \& Shectman, S. A. 2006, AJ, 132, 1714

Rossi, S., Beers, T. C., Sneden, C., Sevastyanenko, T., Rhee, J., \& Marsteller, B. 2005, AJ, 130, 2804

Samus, N. N., et al., General Catalog of Variable Stars, Vizier On-Line Data Catalog

Smith, H. A., RR Lyrae Stars, Cambridge Univ. Press (Cambridge)

Smolinski, J. P., Lee, Y. S., Beers, T. C., et al. 2011, AJ, 141, 89

Sneden, C. A. 1973, PhD thesis, Univ. Texas at Austin

Sneden, C., Cowan J. J., \& Gallino, R. 2008, ARA\&A, 46, 241

Spite, M., Caffau, E., Bonifacio, P., et al. 2013, A\&A, 552, 107

Stancliffe, R, J., Glebbeek, E., Izzard, R. G., \& Pols, O. R. 2007, A\&A, 464, L57

Stancliffe R. J., \& Glebbeek, E. 2008, MNRAS, 389, 1828

Stancliffe, R. J. 2009, MNRAS, 394, 1051

Stancliffe, R. J., Kennedy, C. R., Lau, H. H. B., \& Beers, T. C. 2013, MNRAS, 435, 698

Tominaga, N., Umeda, H., \& Nomoto, K. 2007, ApJ, 660, 516

Tominaga, N., Iwamoto, N., \& Nomoto, K. 2013, ApJ, in press (arXiv:1309.6734)

Umeda, H., \& Nomoto, K. 2003, Nature, 422, 871

Vivas, A. K., Zinn, R., Abad, C., et al. 2004, AJ, 127, 1158

Vivas, A. K., Jaffé, Y. L., Zinn, R., Winnick, R., Duffau, S., \& Mateu, C. 2008, AJ, 136, 1645

Wallerstein, G., Kovtyukh, V., \& Andreivsky, S. 2009, ApJ, 692, L127 